\title{
Glucocorticoids in the treatment of early and late RA
}

\section{J W J Bijlsma, M Boers, K G Saag, D E Furst}

\section{A little GC, like a glass of wine, may benefit many people, whereas a high dose of $G C$, like a bottle of wine, is harmful to all}

\begin{abstract}
$\Delta{ }_{\text {American }}^{\mathrm{t}}$ the 2003 meeting of the Rheumatology (ACR) a debate on the advantages and disadvantages of glucocorticoids (GCs) in the treatment of early and late rheumatoid arthritis (RA) was held, with some authors putting the case for, and others the case against, such treatment (presentations now available online: http:// www.rheumatology.org). Some new data emerged, and this paper summarises the arguments and the existing and new data.
\end{abstract}

\section{HISTORY}

Hench was awarded the Nobel prize in 1950 for the discovery of GCs and their effect in established RA. However, subsequently disillusionment with GCs set in, caused by the rapid appearance of unacceptable side effects of long term high dose treatment, and loss of efficacy at lower dosing. The dogma became that treatment with systemic GCs caused only temporary symptomatic relief, led to habituation with danger of ever increasing doses necessary to maintain effect, and that chronic treatment universally caused unacceptable side effects. Therefore, such treatment was often only considered as a last resort. An associated idea was that RA was in most cases a benign disease, which, although incurable, caused significant disability in only a minority of cases. The combination of these ideas caused most rheumatologists to limit treatment to traditional schemes that emphasised rest, lifestyle adjustment, non-steroidal anti-inflammatory drugs (NSAIDs), and spa treatment. In unresponsive cases antirheumatic drugs such as intramuscular gold were advised. A paradigm shift was initiated by Wilske and Healey, who argued that the pyramid should be inverted and aggressive treatment should be started early. ${ }^{1}$ This was based on the appearance of long term outcome studies that recorded the dismal prognosis of many patients when followed up for a long enough time. With this shift, the door was opened a crack to allow re-examination of the old dogma.

\section{MECHANISMS, USE, AND DOSAGES}

To better understand published reports on the effects of GCs in RA, it is important to recognise two different aspects of the disease process. The first aspect is clinical symptoms of inflammation, which may be inhibited by NSAIDs. This process is partly due to infiltration of the synovial tissue by lymphocytes. The second aspect is erosive progression of the disease, which can be inhibited by disease modifying antirheumatic drugs (DMARDs) such as methotrexate and tumour necrosis factor $\alpha$ blockers. This process is partly due to synovial infiltration by macrophages. Both processes are closely linked, but partially independent and both processes may respond differently to different interventions. GCs seem to influence the inflammatory process especially during the first months of treatment, while effects on erosions become evident after a more prolonged period of time.

\section{"Glucocorticoids act on the inflam- matory process immediately and on erosions only after a longer period of time"}

GCs have a profound effect on the immune system, interfering with the cytokine network and inflammatory enzymes, and with adhesion molecules, permeability factors, cellular function, and survival. GCs are still the most effective anti-phlogistic and immune suppressive substances known. ${ }^{2}$ In early RA, and even in some patients before they develop RA, an insufficiency of the hypothalamic pituitary adrenal (HPA) axis has been documented. Discussion is still continuing as to whether or not this is the "chicken or the egg". In any case it is evident from many studies that cortisol levels found in patients with RA are insufficient to control the continuing inflammatory process. ${ }^{3} 4$

In America up to $75 \%$ of patients with RA use glucocorticoids regularly, concomitantly with other drugs. ${ }^{56}$ In
Europe the percentage is around 25\%, although the role of GCs was reassessed in Europe during the past decade. ${ }^{7}$ In the third world, GC use is very common, as they are one of the only cheap and widely available drugs; they are often sold over the counter. ${ }^{8}$ The official views (as expressed in textbooks on both sides of the Atlantic) are similar and consistent in their opposition to GC treatment. The 2002 update of the guidelines for the management of rheumatoid arthritis of the ACR states: "Low dose oral glucocorticoids $(10 \mathrm{mg}$ or less of prednisone daily) are highly effective at relieving symptoms in patients with active RA. A patient disabled by active polyarthritis may experience marked and rapid improvement in functional status within days following initiation of glucocorticoids. Frequently, disabling synovitis recurs when glucocorticoids are discontinued, even in patients who are receiving combination therapy with one or more DMARDs. Therefore, many patients with RA are functionally dependent on glucocorticoids and continue them long term". ${ }^{\prime \prime}$

What is considered to be a low dose of GCs? In patients with active RA participating in most clinical trials, a mean dosage of glucocorticoids of the order of $7 \mathrm{mg} /$ day is found. ${ }^{5-7}$ In descriptive cohort studies of patients with longstanding RA many patients have less active disease and the mean dosage of glucocorticoids is of the order of 3-5 mg/day, implying that some patients do very well on even a very low dose of GCs. Recently, a consensus meeting on standardisation of the nomenclature of GCs in rheumatic diseases was held. In the meeting report definitions for the dosage, based on pathophysiological and pharmacokinetic data, were suggested: "low dose" was defined as $7.5 \mathrm{mg}$ or less prednisone equivalent a day, a "medium dose" as 7.5-30 mg daily, and a "high dose" was defined as above $30 \mathrm{mg}$ daily. ${ }^{10}$

\section{DOGMA AND FACTS: EFFICACY} Clinical signs of inflammation

It is astonishing to note the paucity of published data that can stand up to critical assessment by current standards. In 1997, a Cochrane review concluded on the basis of seven studies and a total of 253 patients: "Although our findings are based on very few controlled studies, they generally uphold the widely held belief that corticosteroids, when administered for periods of approximately 6 months, are effective for the treatment of $\mathrm{RA}^{\prime \prime} .{ }^{11}$ This conclusion supports the widespread use of GCs, even though guidelines are more negative. 
More recent trials and follow up studies have added important data to our still limited understanding. One hundred and one patients with established RA co-treated with conventional DMARDs were included in a placebo controlled trial that studied $30 \mathrm{mg}$ prednisone daily tapered to $7.5 \mathrm{mg}$ in three weeks, and further tapered according to clinical activity. ${ }^{12}$ More rapid onset of effect in the GC group was found, but the difference between treatment groups was lost after a few months, although effects on Health Assessment Questionnaire (HAQ) and grip strength persisted. The Utrecht group performed a placebo controlled trial of $10 \mathrm{mg}$ prednisone as monotherapy in early $(<1$ year), DMARD naive patients (total, $\mathrm{n}=81$ ). ${ }^{13}$ The duration of the trial was two years; rescue sulfasalazine was allowed after six months, and more than half of the patients in each group started this drug.

\section{"Cochrane review supports the use of GCs, though guidelines are more negative"}

At six months, improvement in disease activity was more rapid in the GC group, despite lower use of intra-articular injections and NSAIDs. However, these differences were no longer significant at one year and later, though the use of NSAIDs and intra-articular injections stayed lower in the GC group. Together with very similar results from the $\mathrm{UK}^{14}$ one can conclude that low to medium dose prednisolone (up to $10 \mathrm{mg}$ /day) added to other DMARDs leads to rapid clinical response that in general does not persist beyond six months.

\section{Radiographic damage}

From the late 1950s onwards studies have pointed to an effect on radiographic damage. Although the early studies were well designed, the results are not so easily summarised because scoring was not standardised. A Cochrane review is currently underway; it will include over 700 patients in about 10 studies. ${ }^{12-14}$ The overwhelming signal is that GCs retard radiological progression. One of the studies included is the COBRA trial, ${ }^{15}$ for which data on long term follow up were recently published. ${ }^{16}$ This trial compared the combination of step-down prednisolone $(60 \mathrm{mg} /$ day down to $7.5 \mathrm{mg} /$ day in seven weeks), methotrexate, and sulfasalazine with sulfasalazine alone: clinical benefits were no longer significant after GCs were stopped, but differences in damage progression were evident up to one year after stopping GCs. It must be emphasised that this was a trial with an active treatment control group and that the patients in this group responded adequately to sulfasalazine as expected. In the long term follow up of COBRA (mean 4.5 years) the yearly rate of progression remained significantly and relevantly lower in the combination group (5.4 Sharp points per year $v 8.6$ in the sulfasalazine group) despite equal treatment, disease activity, and HAQ in both groups. The fact that the progression lines are not parallel (suggesting the initial advantage is maintained) but actually diverge suggests true disease modification of the combination schedule, with GCs in this dose acting either as a specific disease modifier, or as the agent working generically by inducing the rapid initial suppression of disease activity. In the Utrecht study, the only recent study in which GCs were used as monotherapy, a significant decrease in radiographic progression was also seen in the patients, starting at six months. ${ }^{13}$ Comparable data were reported in the UK study in patients with RA in whom GCs were added to other antirheumatic treatment. ${ }^{14}$ In conclusion, low dose GCs clearly decrease radiological progression.

\section{Efficacy summary}

GCs are still the most effective antiphlogistic and immune suppressive substances known. When given for periods of about six months, GCs are effective in reducing signs and symptoms of inflammation in RA; after that time the symptomatic effect wanes when GCs are used as co-medication with DMARDs, though co-medication of NSAIDs, use of intra-articular injections, and other treatments are lower when GCs are continued. Apart from this symptomatic effect, it has now become clear that GCs can reduce the progression of erosions in patients with RA, an effect that even seems to persist when GC treatment is stopped.

\section{DOGMA AND FACTS: TOXICITY}

There is no question that GCs, especially in high doses and continued for longer periods, can cause devastating morbidity. Although the textbooks are full of the "well known" side effects of GCs, we actually know little of their incidence, prevalence, and true impact; and almost nothing about the relation of these effects with different dosing schedules. The quality of evidence on toxicity is even less than that for efficacy. Table 1 lists the toxicities typically associated with chronic GC use. Potential side effects can be divided into those that are possibly preventable and ones that are largely idiosyncratic and typically not preventable. Although observational studies of serious GC adverse effects (AEs) identify fractures and ocular toxicity as among the most prevalent, ${ }^{17}$ patients often worry more about less serious, albeit more apparent, sequelae such as cushingoid facies, skin thinning, and ecchymoses. Catabolic effects on the skin during systemic GC treatment include cutaneous atrophy, resulting from GC effects on keratinocytes and fibroblasts. Decreased vascular structural integrity is probably a key determinant of purpura and easy bruisability in GC treated patients. ${ }^{18}$ These effects were reported to affect over $5 \%$ of those exposed to $5 \mathrm{mg}$ or more prednisone equivalent for one year or longer. ${ }^{19}$ At the lower dose range of GCs (below the physiological threshold) commonly used by American doctors to manage RA, change in personal appearance is an uncommon outcome. However, the traditionally assumed physiological threshold (about $7.5 \mathrm{mg} /$ day of prednisone) may be overestimated ${ }^{20}$; thus, even very low doses may exceed it and lead to these AEs.

\section{Bone}

Of all serious AEs attributed to GCs, bone loss leading to fractures provokes the most trepidation among knowledgeable doctors. Long term follow up of women in the large Arthritis and Medical Information System (ARAMIS) cohort identified a risk of about $33 \%$ of having a fracture after five years of follow up on an average GC dose of only $8.6 \mathrm{mg} /$ day. ${ }^{21}$ In a large Norwegian cohort current GC use was associated with loss of bone mineral density (BMD) at both the total hip (adjusted odds ratio $(\mathrm{aOR})=2.6$ ) and spine $(\mathrm{aOR}=2.7))^{22}$ Although factors other than BMD predispose to fracture risk in these patients, BMD of both the spine and hip is a significant independent predictor of vertebral fracture among GC users. ${ }^{23}$ Negative effects on bone formation, in contrast with heightened bone resorption, appear to be most central to GC induced osteoporosis. $^{24}$

\section{"Some data suggest that GCs may even benefit the bones of patients with RA $^{\prime \prime}$}

Despite this pathogenic rationale and accumulating evidence from observational studies on GC risk to bone, some data suggest that GCs may actually benefit bones in patients with RA.25 This benefit may be brought about by decreasing the disease activity, promoting beneficial weightbearing activity and a reduction in proinflammatory cytokines deleterious to bone. ${ }^{26}$ These dissenting opinions on the toxicity of 
Table 1 Potential glucocorticoid toxicity

\begin{tabular}{ll}
\hline Potentially preventable & Not preventable \\
\hline Acne vulgaris & Accelerated atherosclerotic vascular disease \\
Congestive heart failure and fluid retention & Cataracts \\
Diabetes mellitus & Mood change \\
Glaucoma & Myopathy \\
Hypertension & Osteonecrosis \\
Osteoporosis & Serious infection \\
Peptic ulcer disease & Skin bruising/thinning \\
Sleep disturbances and memory effects & Weight gain \\
\hline
\end{tabular}

glucocorticoids to bone have been strengthened by mixed and inconclusive results from recent large randomised controlled trials (RCTs) of GC use in RA. It is important to note, however, that to date GC RCTs have been significantly underpowered to detect even modest differences in fractures between patients receiving and not receiving GCs. In addition to doctors' concerns and beyond the disability, morbidity, and attributable mortality of fractures, patients receiving GCs greatly fear this complication. When the preferences of a patient with RA for GC sequelae were evaluated using hypothetical decision scenarios, serious fractures were rated as the worst of all possible GC complications (preference value of 0.55; values are anchored at $0=$ death and $\mathrm{l}=$ current health). ${ }^{27}$ Although the osteoporosis complications of GC use are a major reason for questioning their role in RA management, the advent of effective and safe prevention for GC induced osteoporosis has partially ameliorated this problem. The ACR and a number of other specialty based consensus groups have issued guidelines or position statements advocating a much more aggressive approach to GC induced osteoporosis prevention..$^{28}$

In addition to periodic bone mass measurement, American, Canadian, and Australian guidelines also advocate aggressive use of aminobisphophonate drugs such as alendronate or risedronate. Although the indications for the use of bisphosphonate vary based on these different guidelines, patients expected to require prolonged supraphysiological GC doses, those with existing low bone mass ( $\mathrm{T}$ score $<-1.0$ in some and less than -1.5 in other guidelines), and postmenopausal women are high risk groups that should receive bisphosphonates sooner rather than later. Other therapeutic options such as calcitonin, oestrogen, testosterone, and possibly, thiazide diuretics (early in the course of GC use) have more limited scientific support. Despite the clear efficacy of bisphosphonates to significantly increase bone mass and lower vertebral fracture risk (study designs limited by sample size), bisphosphonates do not cure GC induced osteoporosis. Furthermore, therapeutic costs and the need for increasing polypharmacy limit enthusiasm for a combined approach of GCs and bisphosphonates in RA. ${ }^{29}$

In summary, although some treatment is available to prevent bone complications of GCs, these complications are still of concern at even low doses of GCs.

Another less common bone complication of GCs is osteonecrosis (ON). Although ON may have a similar pathogenesis to that of GC induced osteoporosis, it is very unusual among patients treated with lower doses, such as in RA. An incidence of ON of $<3 \%$ was seen at replacement doses and clinically significant $\mathrm{ON}$ is seldom reported in patients with RA receiving GC doses below $20 \mathrm{mg} /$ day. ${ }^{30}$

\section{Gastrointestinal}

Gastrointestinal (GI) AEs of GCs alone are less severe than those seen when non-selective NSAIDs are used. However, when GCs are used in combination with NSAIDs, a synergistic increased risk of GI AEs is observed. In a Medicare database 1400 patients with different inflammatory diseases receiving GC treatment were evaluated: the relative risk of a peptic ulcer or GI bleed was 2.0, but multivariate analysis showed that concomitant use of NSAIDs explained this increased risk, ${ }^{31}$ indicating that GCs alone have no increased risk for GI events. In an observational study of 566 patients with RA using steroids (mean duration of use 5.1 years; mean prednisone dose $6.84 \mathrm{mg} /$ day) compared with 253 controls followed up over slightly more than eight years, even low dose prednisone $(<5.0 \mathrm{mg} /$ day) was associated with an increased risk of GI ulcerations, with an OR of 1.8 after a mean of 1.5 years of use. ${ }^{32}$ This OR increased slightly, up to an OR of 2.1 when GCs were used for more than five years.

\section{Eye}

Similar to bone disease, cataracts are ubiquitous with prolonged GC treatment. Posterior subcapsular cataracts, although less common than senile or cortical cataracts in a population, are the type of cataracts most typically associated with GC treatment. ${ }^{33}$ Biochemically, GC-lysine adducts causing lens particles to form, and inhibition of the sodium-potassium pump increasing the water content of the lens may explain this complication. Although there have been few comprehensive analyses of this risk, posterior subcapsular cataracts may develop in as few as two years on as little as $5 \mathrm{mg} /$ day of prednisone. Of interest, a population based study has also detected a $>2.5$ fold increased risk of cortical cataracts in GC users compared with non-users. ${ }^{34}$ Inhaled GCs have been associated with increased risk of cataracts, emphasising a potential effect with even very low doses. ${ }^{35}$ Overall, limited data on glucocorticoids and cataracts seem to support the association of GCs and cataracts, but these data also highlight the need for more research into this toxicity.

\section{Cardiovascular}

Although acute hypertension and lipoprotein perturbations with GCs may ultimately result in a higher risk of cardiovascular disease, ${ }^{36} 37$ investigation into this topic is particularly confounded by RA disease activity. An association of RA with a higher risk of cardiovascular disease may relate to a direct deleterious impact of prolonged inflammation on cardiovascular disease. Because antiinflammatory agents may lower the risk of cardiovascular disease, long term data are needed to determine whether a GC effect on atherosclerotic disease is independent of RA. Prior data from retrospective cohorts have attributed GC use to heightened overall mortality, ${ }^{38}$ although confounding by indication is problematic in these types of observational studies. Recent data from cohorts show that RA disease activity alters the blood lipid profile unfavourably, and treatment (including GC treatment) can reverse these changes. ${ }^{39}$

\section{Glucose metabolism}

It takes only a few weeks before hyperglycaemia can occur when using GCs and there is both a dose-response and inverse duration effect for this end point. At doses of 1-7.9 mg prednisone daily, the OR for hyperglycaemia is 1.8 ; this rises progressively to an OR of 7 when the dose of prednisone is $25 \mathrm{mg}$ daily or more. The OR of requiring treatment for hyperglycaemia is 1.7 during the first 45 days of corticosteroid use, falls to 1.3 between 46 and 90 days, and is merely 1.1 beyond that point. ${ }^{40}$ As this is based on an observational cohort study, a decrement in risk over time is logical, as GCs will be stopped if hyperglycaemia is found and hypergly- 
caemia was found early in GC use in this study, probably because serum glucose was monitored. The development of overt diabetes in patients using low dose GCs is rare.

\section{Infections}

In an American evaluation of 566 patients with RA receiving GCs, a $22.4 \%$ incidence of infections versus $13 \%$ in the controls was observed. ${ }^{32}$ The OR for an infection was 1.72 when steroids were used for six months or longer. This result, however, contrasts with the results from a review on infections complications in patients taking GCs. ${ }^{41}$ In five studies quoted in the review, there was no increase in infection rate among rheumatic patients given up to $8 \mathrm{mg}$ GCs daily, although the duration of use of the steroids in those studies was not delineated. Another review stated that GC doses $\geqslant 10 \mathrm{mg}$ daily (total dose $>700 \mathrm{mg}$ ) for at least 3-4 weeks were needed before there was an increased incidence of infection. ${ }^{19}{ }^{42}$ Infections are probably increased in GC users, although relatively insignificantly for patients receiving low dose GCs for RA.

\section{HPA axis}

There is little doubt that higher doses of prednisone over long periods can cause HPA axis suppression and many published reports attest to this effect. ${ }^{43}$ On the other hand, the best data relating to very low doses of prednisone come from a meta-analysis comparing the effects of intranasal steroid preparations, which used oral prednisone as a comparator. ${ }^{43}$ In an analysis of 13 studies and using 800 am serum cortisol as a measure of HPA suppression, it appears that doses $<8 \mathrm{mg} /$ day prednisolone (the exact dose is hard to discern from the figure) will suppress 800 am cortisol. These data show that low doses of GCs, in the range of the doses often used when treating RA, can regularly cause HPA suppression, although the duration of such treatment required for such an effect to occur is not explicitly defined. To make these data more difficult to interpret, it has been suggested that RA itself has an influence on the HPA axis.

\section{Mental functioning}

The doses of prednisone used to treat RA have not usually been associated with steroid psychosis, so this aspect of the side effects of GC in early RA will not be considered further here ${ }^{44}$ However, GCs do seem to have an effect on neuronal death. ${ }^{45}$ When GCs are used, translocation of pre-existing glucose cell surface receptors to the intracellular environment seems to occur, resulting in 25 $30 \%$ inhibition of glucose transport into cells. This accelerates the usual decline in ATP concentrations after ischaemia or hypoglycaemia. This, in turn, decreases the ability to inhibit neuronal insults. This theoretical construct has been carried into a study of normal volunteers given $10 \mathrm{mg}$ oral hydrocortisone in a single blind study. ${ }^{46}$ Hippocampal dependent functions such as declarative memory and spatial thinking, as well as non-hippocampal dependent measures such as procedural thinking were tested. The hippocampal dependent functions were found to be impaired. In another test of mental functioning, paired association word recall and consolidation of verbal learning (hippocampal dependent functions) were impaired at the time of hydrocortisone infusions and four days later. ${ }^{47}$ These experiments in normal volunteers were later tested in patients with RA who had been receiving GCs for more than one year and compared with the results for controls. ${ }^{48}$ Once again, hippocampal functioning (recall of explicit facts) was impaired, while non-hippocampal functioning (procedural memory) was not.

\section{Toxicity summary}

An increasing number of clinical trials comparing GC users with non-users inform us about the short and moderate term AEs in GC users. There are credible data that even low dose GCs given for weeks to months can have significant toxicity in numerous areas, including GI ulcerations and bleeding when given together with NSAIDs, an increased incidence of infections, an increased incidence of hyperglycaemia, a predisposition to glaucoma, suppression of the HPA axis, abnormalities in recall and mental functioning, and skin ecchymoses and thinning. Despite this increasing knowledge, longer term data are badly needed to assess the impact of this most commonly used treatment. Bone loss and cataracts are the complications most well validated, whereas other long term AEs need further investigation. Despite the fact that GCs improve functional status and reduce radiographic erosions, it is imperative that we place their long term side effects into the therapeutic equation in order to determine their appropriate place in our treatment armamentarium.

\section{CONCLUSION}

There is thus clear evidence that GCs improve physical function and reduce progression of joint damage in the treatment of RA. However, it cannot be emphasised enough that the cautious use of GCs in patients with longstanding RA should be weighed and balanced for the individual patient. A take home message might be: "A little GC, like a glass of wine, may benefit many (some $-(\mathrm{sic}))$ people, whereas a high dose of GC, like a bottle of wine is harmful to all" ${ }^{49}$ In both cases long term intake might be harmful.

Ann Rheum Dis 2003;62:1033-1037

\section{Authors' affiliations}

J W J Biilsma, Department of Rheumatology and Clinical Immunology (F02.127), University Medical Centre Utrecht, P.O. Box 85500, 3508 GA Utrecht, The Netherlands

$M$ Boers, Department of Clinical Epidemiology and Biostatistics, VU Medical Centre,

Amsterdam, The Netherlands

K G Saag, Division of Clinical Immunology and Rheumatology, University of Alabama at Birmingham, Birmingham, AL, USA

D E Furst, Department of Rheumatology, University of California, Los Angeles, CA USA

Correspondence to: Professor J W J Biilsma; i.w.j.bij|sma@azu.nl

\section{REFERENCES}

1 Wilske KR, Healey LA. Remodeling the pyramida concept whose time has come. J Rheumatol 1989;16:565-7.

2 Bijlsma JWJ, Straub RH, Masi AT, Lahita RG Cutolo M. Neuroendocrine immune mechanisms in rheumatic diseases. Trends Immunol 2002;23:59-61.

3 Dekker JC, Geenen R, Godaert GLR, Bijlsma JWJ. Diurnal rhythm of salivary cortisol in patients with RA of recent onset. Arthritis Rheum 2000;43:465-7.

4 Cutolo M, Foppiani L, Prete C, Ballarino P, Sulli A, Villagio $B$, et al. Hypothalamic-pituitaryadrenocortical axis in premenopausal rheumatoid arthritis patients not treated with glucocorticoids. I Rheumatol 1999:26:282-8.

5 Weinblatt ME, Kremer JM, Coblyn JS, Maier AL, Helfgott SM, Morell M, et al. Pharmacokinetics, safety and efficacy of combination treatments with methotrexate and leflunomide in patients with active RA. Arthritis Rheum 1999;42:1322-8.

6 Moreland LW, Schiff MH, Baumgarten SW, Tindall EA, Fleischman RM, Bulpitt KJ, et al. Etanercept therapy in RA: a randomized controlled trial. Ann Intern Med 1999; 130:478-86.

7 Smolen JS, Kalden JR, Scott DL, Rozman B, Kvier TK, Larsen A. Efficacy and safety of leflunomide compared with placebo and sulphasalazine in active RA: a double-blind, randomised, multicentre trial. Lancet 1999:353:259-66.

8 Kalla AA, Tikly M. Rheumatoid arthritis in the developing world. Best Pract Res Clin Rheumatol 2003; 17:863-75.

9 ACR Subcomittee on Rheumatoid Arthritis Guidelines. Guidelines for the management of RA: 2002 update. Arthritis Rheum 2002;46:328-46.

10 Buttgereit $F$, Da Silva JAP, Boers $M$ Burmester GR, Cutolo M, Jacobs J, et al. Standardised nomenclature for glucocorticoid dosages and glucocorticoid treatment regimens: current questions and tentative answers in rheumatology. Ann Rheum Dis 2002:61:718-22.

11 Criswell LA, Saag KG, Sems KM, Welch V, Shea B, Wells G, et al. Moderate-term, low-dose corticosteroids for rheumatoid arthritis. Cochrane Database Syst Rev 2000:CD001158.

12 Hansen M, Podenphant J, Florescu A Stoltenberg M, Borch A, Kluger E, et al. A randomised trial of differentiated prednisolone treatment in active rheumatoid arthritis. Clinical benefits and skeletal side effects. Ann Rheum Dis 1999:58:713-18. 
13 Van Everdingen AA, Jacobs JWG, Siewertsz van Reesema DR, Bijlsma JWJ. Low dose prednisone therapy for patients with early active rheumatoid arthritis; clinical efficacy, disease modifying properties and side effects: a randomized doubleblind placebo-controlled clinical trial. Ann Intern Med 2002; 136:1-12.

14 Kirwan JR. Arthritis and Rheumatism Council Low-Dose Glucocorticoid Study Group. The effect of glucocoricoids on joint destruction in rheumatoid arthritis. N Engl J Med 1995:333:142-6.

15 Boers M, Verhoeven AC, Markusse HM, van de Laar MA, Westhovens R, van Denderen JC, et al. Randomised comparison of combined step-down prednisolone, methotrexate and sulphasalazine with sulphasalazine alone in early rheumatoid arthritis. Lancet 1997;350:309-18.

16 Landewe RB, Boers $M$, Verhoeven AC, Westhovens $R$, van de Laar MA, Markusse HM, et al. COBRA combination therapy in patients with early rheumatoid arthritis: Long-term structural benefits of a brief intervention. Arthritis Rheum 2002:46:347-56

17 Saag KG, Koehnke R, Caldwell JR, Brasington R, Burmeister LF, Zimmerman B, et al. Low dose long-term corticosteroid therapy in rheumatoid arthritis: an analysis of serious adverse events. Am J Med 1994;961:1 15-23.

18 Davies GF. Adverse effects of corticosteroids: II Systemic. Clin Dermatol 1986;4:161-9.

19 Caldwell JR, Furst DE. The efficacy and safety of low-dose corticosteroids for rheumatoid arthritis. Semin Arthritis Rheum 1991;21(:1-11.

20 Esteban N, Loughlin T, Yergey AL, Zawadzki JK Booth JD, Winterer JC, et al. Daily cortisol production rate in man determined by stable isotope dilution/mass spectrometry. $J$ Clin Endocrinol Metab 1991;72:39-45.

21 Michel BA, Bloch DA, Fries JF. Predictors of fractures in early rheumatoid arthritis. J Rheumato $1991 ; 18: 804-8$.

22 Haugeberg G, Orstavik RE, Uhlig T, Falch JA, Halse Jl, Kvien TK. Bone loss in patients with rheumatoid arthritis: results from a populationbased cohort of 366 patients followed up for two years. Arthritis Rheum 2002;46:1720-8.

23 Cooper C, Coupland C, Mitchell M. Rheumatoid arthritis, corticosteroid therapy and hip fracture. Ann Rheum Dis 1995; 54:49-52.

24 Rubin MR, Bilezikian JP. Clinical review 151: the role of parthyroid hormone in the pathogenesis of glucocorticoid-induced osteoporosis: a re-examination of the evidence. J Clin Endocrinol Metab 2002;87:4033-41.

25 Verhoeven A, Boers M. Limited bone loss due to corticosteroids: a systematic review of prospective studies in rheumatoid arthritis and other diseases. J Rheumatol 1997;24:1495-503.

26 Sambrook PN, Eisman JA, Yeates MG Pocock NA, Eberl S, Champion GD. Osteoporosis in rheumatoid arthritis: safety of low dose corticosteroids. Ann Rheum Dis 1986;45:950-3.

27 Merlino L, Bagchi I, Taylor T, Summner W, Nease R, Saag K. Preference values for corticosteroid-associated adverse health states in 107 rheumatoid arthritis patients. Med Decis Making 1998;18:470.

28 ACR. American College of Rheumatology Ad Hoc Committee on Glucoorticoid-Induced Osteoporosis. Recommendations for the prevention and treatment of glucocorticoidinduced osteoporosis. Arthritis Rheum 2001;44: 1496-503.

29 Saag K, Pisu M. Balancing bones and bucks among new glucocorticoid users. J Rheumatol 2003;301:1-3.

30 Vreden SG, Hermus AR, van Liessum PA, Pieters GF, Smals AG, Kloppenborg PW. Aseptic bone necrosis in patients on glucocorticoid replacement therapy. Neth J Med 1991;39:153-17.

31 Piper JM, Ray WA, Daugherty JR, Griffin MR. Corticosteroid use and peptic ulcer disease: role of nonsteroidal anti-inflammatory drugs. Ann Intern Med 1991;114:735-40.

32 Wolfe F, Furst DE, Lane N, Anderson J, Hawley DJ. Substantial increases in important adverse events follow low dose prednisone therapy of rheumatoid arthritis (RA) [abstract] Arthritis Rheum 1995;38(suppl):S312.

33 Carnahan MC, Goldstein DA. Ocular complications of topical, peri-ocular, and systemic corticosteroids. Curr Opin Opthalmol 2000;11:478-83.

34 Klein BE, Klein R, Lee KE, Danforth LG. Drug use and five-year incidence of agerelated cataracts: the Beaver Dam Eye Study. Opthalmology $2001 ; 108: 1670-4$.

35 Cumming RG, Mitchell P, Leeder SR. Use of inhaled corticosteroids and the risk of cataracts. N Engl Med 1997;337:8-14.

36 Kalbak K. Incidence of arteriosclerosis in patients with rheumatoid arthritis receivinglong-term corticosteroid therapy. Ann Rheum Dis 1972;31:196-200.
37 Jackson SHD, Beevers DG, Meyers K. Does longterm low-dose corticosteroid therapy cause hypertension? Clin Sci 1981;61:381-33s.

38 Wolfe F, Mitchell DM, Sibley JT, Fries JF, Bloch DA, Williams CA, et al. The mortality of rheumatoid arthritis. Arthritis Rheum 1994;37:481-94

39 Boers M, Nurmohamed MT, Doelman CJA Lard LR, Verhoeven AC, Voskuyl AE, et al. The influence of corticosteroids and disease activity on total and $\mathrm{HDL}$-cholesterol in patients with rheumatoid arthritis (RA). Ann Rheum Dis 2003;62:842-5.

40 Gurwitz JH, Bohn RL, Glynn RJ, Monane M, Mogun H, Avorn J. Glucocorticoids and the risk for initiation of hypoglycemie therapy. Arch Intern Med 1994:154(1):97-101.

41 Stuck AE, Minder CE, Frey FJ. Risk of infectious complications in patients taking glucocorticosteroids. Rev Infect Dis 1989; 11:954-63.

42 Aucott JN. Glucocorticoids and infection. Endocrinol Metab Clin North Am 1994;23:655-70.

43 Lipworth BJ. Systemic adverse effects on inhaled corticosteroid therapy: a systematic review and meta-analysis. Arch Intern Med 1999:10:941-55

44 Wolkowitz OM, Reus VI, Canick J, Levin B, Lupien S. Glucocorticoid medication, memory and steroid psychosis in medical illness. Ann N Y Acad Sci 1997:823:81-96.

45 McIntosh L, Sapolsky RM. Glucocorticoids may enhance oxygen radical-mediated neurotoxicity. Neurotoxicity 1996;17:873-82.

46 Kirschbaum C, Wolf OT, May M, Wippich W, Hellhammer DH. Stress- and treatment-induced elevations of cortisol levels associated with impaired declarative memory in healthy adults. Life Sci 1996:58:1475-83.

47 Keenan PA, Jacobson MW, Soleymani RM, Mayes MD, Stress ME, Yaldoo DT. The effect on memory of chronic prednisone treatment in patients with systemic disease. Neurology 1996:47:1396-402.

48 Keenan PA, Jacobson MW, Soleymani RM, Newcomer JW. Commonly used therapeutic doses of glucocorticoids impair explicit memory. Ann N Y Acad Sci 1995;761:400-2.

49 Pincus T, Sokka T, Stein CM. Are long-term very low doses of prednisone for patients with rheumatoid arthritis as helpful as high doses are harmful? Ann Intern Med 2002;136:76-8 